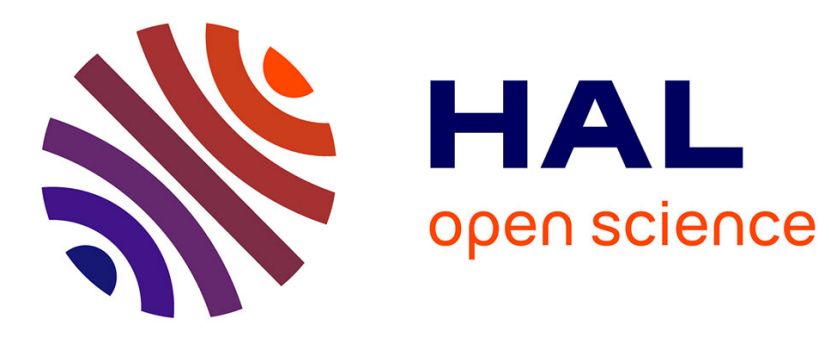

\title{
Lattice modes and plasmonic linewidth engineering in gold and aluminum nanoparticle arrays
}

Dmitry Khlopin, Frédéric Laux, William P. Wardley, Jérôme Martin, Gregory

A. Wurtz, Jérôme Plain, Nicolas Bonod, Anatoly V. Zayats, Wayne Dickson, Davy Gérard

\section{To cite this version:}

Dmitry Khlopin, Frédéric Laux, William P. Wardley, Jérôme Martin, Gregory A. Wurtz, et al.. Lattice modes and plasmonic linewidth engineering in gold and aluminum nanoparticle arrays. Journal of the Optical Society of America B, 2017, 34 (3), 10.1364/JOSAB.34.000691 . hal-01571589

\section{HAL Id: hal-01571589 \\ https://hal.science/hal-01571589}

Submitted on 19 Jul 2018

HAL is a multi-disciplinary open access archive for the deposit and dissemination of scientific research documents, whether they are published or not. The documents may come from teaching and research institutions in France or abroad, or from public or private research centers.
L'archive ouverte pluridisciplinaire HAL, est destinée au dépôt et à la diffusion de documents scientifiques de niveau recherche, publiés ou non, émanant des établissements d'enseignement et de recherche français ou étrangers, des laboratoires publics ou privés. 


\title{
Lattice modes and plasmonic linewidth engineering in gold and aluminum nanoparticle arrays
}

\author{
Dmitry Khlopin ${ }^{1, \dagger}$, Frédéric LauX ${ }^{1, \dagger}$, William P. WARdLey ${ }^{2, \dagger}$, Jérôme Martin ${ }^{1}$, Gregory A. \\ Wurtz $^{2}$, Jérôme Plain ${ }^{1}$, Nicolas Bonod ${ }^{3}$, Anatoly V. Zayats ${ }^{2}$, Wayne Dickson ${ }^{2}$, and Davy \\ GÉRARD ${ }^{1,2, *}$ \\ ${ }^{1}$ Laboratoire de Nanotechnologie et d'Instrumentation Optique, Institut Charles Delaunay, UMR CNRS 6281, Université de Technologie de Troyes, France \\ ${ }^{2}$ Department of Physics, King's College London, Strand, London WC2R 2LS, United Kingdom \\ ${ }^{3}$ CNRS, Aix-Marseille Université, Centrale Marseille, Institut Fresnel, UMR 7249, Campus de Saint Jérôme, 13397 Marseille, France \\ ${ }^{\dagger}$ These authors have contributed equally to this work. \\ *Corresponding author: davy.gerard@utt.fr
}

Compiled December 7, 2016

\begin{abstract}
Lattice modes have been proposed as a means to engineer and control the linewidth and spectral position of optical resonances in arrays of metallic nanoparticles sustaining localized surface plasmon (LSP) resonances. Lattice modes are produced by the interference of LSP-enhanced in-plane scattered light, leading to a Fano-like lineshape with reduced linewidth. In this paper, we study the lattice modes supported by gold and aluminium nanoparticle arrays in the visible and UV, both experimentally and theoretically. The measured and simulated dispersion curves allow us to comprehensively analyze the details of the LSP coupling in the array. We show that when the spectral position of the Rayleigh anomaly, which depends on the period of the array, is slightly blue-shifted with respect to the LSP resonance, the quality factor in the nanoparticle array is significantly increased. We also provide evidence that the formation for the lattice modes, i.e. the coupling between LSPs and the in-plane scattered light, critically depends on the incident light polarization, the coupling efficiency being maximum when the polarization direction is perpendicular to the propagation direction of the grazing wave. The results obtained provide design rules allowing high quality factor resonances throughout visible and ultraviolet spectral ranges, needed for sensing and active nanophotonic applications. (c) 2016 Optical Society of America
\end{abstract}

OCIS codes: (240.6680) Surface plasmons; (050.1950) Diffraction gratings; (160.3900) Metals; (310.6628) Subwavelength structures, nanostructures.

http://dx.doi.org/10.1364/josab.XX.XXXXXX

\section{INTRODUCTION}

The field of plasmonics has been attracting a lot of attention because it allows light control at the nanoscale [1]. In many cases, this relies on localized surface plasmon (LSP) resonances sustained by metal nanoparticles. When illuminated at its resonant frequency, the metal's free electron gas strongly interacts with light yielding a significative enhancement of the electromagnetic field in the vicinity of the nanoparticle. This resonance frequency, $\omega$, critically depends on a number of parameters including the nature of the metal, the size and shape of the nanoparticle, and the refractive index of the surrounding medium. As is the case with any resonant phenomenon, the width of the resonance peak is directly related to the associated losses, including scattering and absorption losses. The resonance width $\Delta \omega$ is often described using the quality factor, $Q$, as a figure of merit, defined as $Q=\omega / \Delta \omega$. Due to intrinsic losses in metals, the quality factor of LSP resonances is usually modest (in the 1-10 range) and mainly depends on the nature of the metal - noble metals such as silver and gold exhibiting better quality factors than the so-called "poor" metals in the visible and near-infrared spectrum. However, poor metals can be valuable plasmonic metals, especially outside the visible spectrum [2]. For instance, aluminum is currently being rediscovered as a relevant material for plasmonics, especially in the ultraviolet (UV) spectrum (see [3] for a review). Actually, the main interest in aluminum as a plasmonic material lies in its broadband nature: apart from a relatively small region around $\lambda=800 \mathrm{~nm}$ (corresponding to the interband transition), aluminum can sustain LSPRs from the deep-UV to the infrared [4,5]. Aluminum nanostructures have been used so far both in the UV, for instance to realize an UV-emitting plasmonic laser [6], and in the visible range - e.g. as a platform for color rendering [7, 8], or for photodetection [9]. 
However, the amount of losses in the visible and IR regions is significantly higher than for noble metals, leading to relatively poor quality resonances.

The quality factor of LSP resonances can be improved in geometries where plasmonic particles are arranged in a periodic array, so that electromagnetic coupling between scatterers taking place due to diffractive effects leads to the emergence of so-called lattice modes (see Ref. [10] for a review). These diffracting lattice modes, sometimes called surface lattice resonances, are generally observed near a Rayleigh anomaly of the array. Rayleigh anomalies correspond to the appearance (or disappearance) of diffracted orders, and are associated with a grazing wave propagating in the plane of the array [11]. Lattice modes can be described via a Fano-type process involving a broad resonance (the LSP resonance) and a discrete state associated to the light scattered in the plane of the array at the Rayleigh anomaly position [12]. This coupling yields two hybrid states sharing the properties of its contributing components. The lattice mode is one of these hybrid states, located close to the Rayleigh anomaly position, that offers an enhanced scattering cross-section, based on the LSP contribution, while also demonstrating a high quality factor [12-14]. This effect was predicted as early as 1985 in the context of rough surfaces for surface-enhanced Raman scattering $[15,16]$, before being rediscovered almost 20 years later for one-dimensional arrays of silver nanoparticles [17]. Later, lattice modes in two-dimensional gold nanoparticles arrays were shown to significantly improve the finesse of the resonance [14, 18-24], finding applications in metal-enhanced fluorescence [25], non-linear optics [26] and the realization of plasmon-based lasers [27]. Aluminum nanoparticle arrays have been shown to enhance and redirect the emission of nearby dye molecules, with potential applications including solid-state lighting [28, 29]. Recently, a plasmonic sensor based on the lattice modes sustained by a one-dimensional aluminum grating has been demonstrated [30]. It should also be emphasized that lattice modes can be observed in different periodic systems, such as hole arrays [31, 32], arrays of magnetic nanoparticles [33] and $\mathrm{SiC}$ nanocylinders arrays at midinfrared wavelengths [34].

In this paper, we report on a comprehensive and comparative study of the lattice modes supported by gold and aluminum gratings in the visible and UV regions. Dispersion plots are experimentally retrieved, allowing us to analyze the coupling between LSPs in the array. In the recent review, [10] the critical role played by the periodicity has been underlined in order to correctly position the Rayleigh anomalies in the spectrum with respect to the LSP and hence observe lattice modes. In the present work, we evidence the role of (i) the dielectric environment of the array and (ii) the polarization of the incident light, showing that the scattering pattern of the individual nanoparticles is the key parameter that controls the formation of the lattices modes. The paper is organised as follows. Section 2 is devoted to the design of our structures using numerical calculations. The role of the dielectric environment is unveiled by plotting the dispersion curves of the arrays. Then in Section 3 we present the experimental methods we used to fabricate and characterize the nanoparticles arrays. Results obtained on gold and aluminum nanoparticles arrays are presented in Sections 4 and 5. Finally, in Section 6 we summarize our results and conclude.

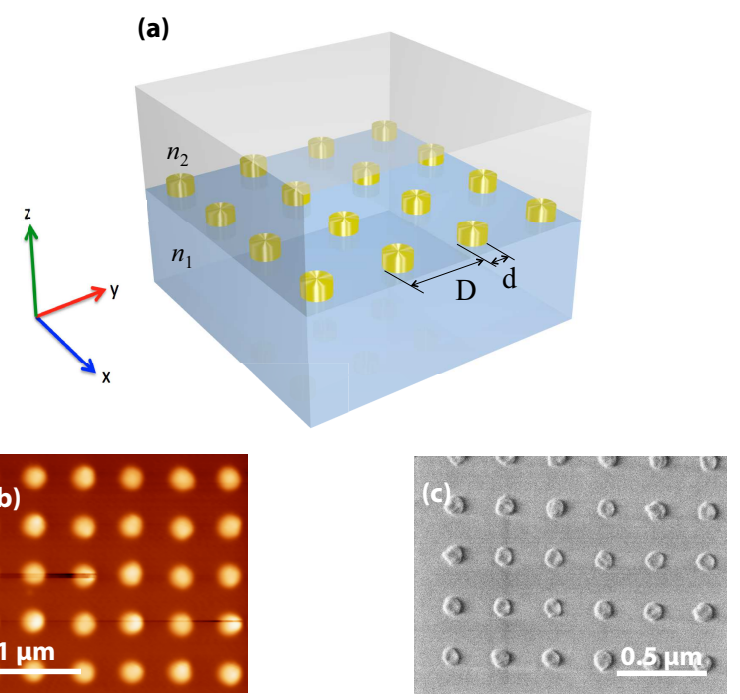

Fig. 1. (a) Schematic of the considered geometry: an array of metal nanoparticles deposited onto a dielectric substrate of refractive index $n_{1}$. The superstrate can be air or a dielectric with refractive index $n_{2}$. (b) AFM image of a square array of gold nanodisks with $D=480 \mathrm{~nm}, d=160 \mathrm{~nm}$ and $h=50$ $\mathrm{nm}$. (c) SEM image of an array of aluminum nanodisks with $D=300 \mathrm{~nm}, d=120 \mathrm{~nm}$ and $h=50 \mathrm{~nm}$.

\section{LINEWIDTH ENGINEERING WITH LATTICE MODES}

\section{A. Design and modelling}

The structure under study is shown in Fig. 1a. It consists of a square array of nanoparticles (nanodisks) deposited onto a dielectric substrate with refractive index $n_{1}$. The period of the array is denoted $D$, the diameter of the nanodisks is $d$ and their thickness is $h$. Two cases were considered, depending on the refractive index $n_{2}$ of the superstrate (upper medium). In the asymmetric configuration, the upper medium is simply air $\left(n_{2}=1\right)$. In the symmetric case, a superstrate whose refractive index is close to that of the substrate (e.g. a polymer layer) is added, so that the nanoparticles are effectively located inside a homogeneous environment.

To design the structures, we performed numerical modelling based on the finite difference time domain (FDTD) method [35]. 3D modelling was used, with PML as boundary conditions on the top and bottom sides of the simulation box, while periodic boundary conditions are applied on the remaining four sides. An adaptive mesh was used to precisely model the shape of the nanoparticle. Refractive indices were taken from Ref. [36] and [37]. The incident light is a plane wave, impinging from the substrate side. Oblique incidence on the periodic structure was described using the approach presented in Ref. [38]. It is worth noting that due to the peculiar nature of the investigated lattice resonances, a special attention must be paid to the PML delimiting the simulation box. Lattice modes are high Q-factor (long-lived) resonances with a significant spatial extension and propagates in a direction that is parallel to the PML layers. This may yield undesired reflections on the PMLs leading to a wrong estimation of the resonance width. Here, we used the stretched coordinates PMLs as implemented in [35] and performed exten- 


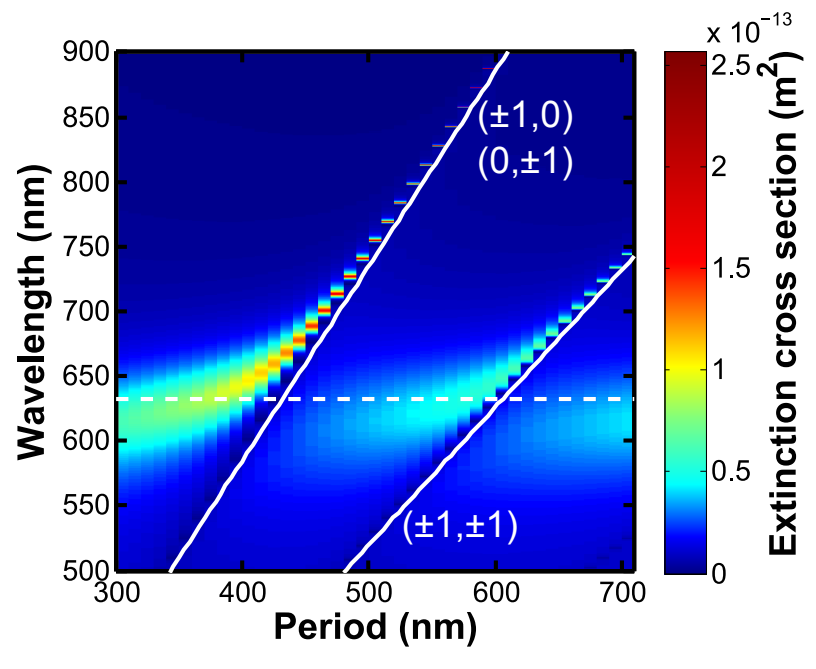

Fig. 2. Normal incidence extinction cross-section spectrum calculated with FDTD for a square array of gold nanodisks with diameter $d=80 \mathrm{~nm}$ and thickness $h=50 \mathrm{~nm}$, for different lattice periods and in a homogeneous dielectric environment $\left(n_{1}=n_{2}=1.5\right)$. Solid lines show the theoretical position of Rayleigh anomalies, and the dotted horizontal line is the position of the dipolar resonance from the isolated gold nanodisk.

sive convergence tests.

\section{B. Effect of the dielectric environment}

We focus our attention on gold nanoparticles arrays first. Fig. 2 shows a map of the normal incidence extinction cross-section $\sigma_{\text {ext }}$, as calculated using FDTD, for a square array of gold nanodisks with diameter $D=80 \mathrm{~nm}$ and thickness $h=50 \mathrm{~nm}$ plotted for different lattice periods and wavelengths. Here we consider a symmetric dielectric environnement, as the refractive indices of the substrate and superstrate are set to $n_{1}=n_{2}=1.5$. Experimentally, this corresponds to a situation where the nanoparticles are deposited onto a substrate and subsequently covered with an index-matching superstrate. For shorter periods, the resonance position of the array, observed as a maximum in the extinction, is very close to the LSP resonance of an isolated particle (dotted horizontal line) but as the period increases, the resonance position red-shifts and simultaneously sharpens significantly. Moreover, the lineshape is also modified, from a relatively symmetric shape to a Fano-like lineshape (see the resonance near $\lambda=750 \mathrm{~nm}$ in Fig. 3, insert). To understand this behavior, we superimposed on the dispersion diagram the theoretical position of the Rayleigh anomalies. According to grating theory, in the most general case of a square grating with period $D$ sitting on the interface between two media with refractive indices $n_{1}$ and $n_{2}$, the position of the Rayleigh anomalies is given by:

$$
\lambda_{m, p}=D \frac{\sqrt{n_{2}^{2}\left(m^{2}+p^{2}\right)-n_{1}^{2} p^{2} \sin ^{2} \theta} \pm m n_{1} \sin \theta}{m^{2}+p^{2}}
$$

where $\theta$ is the angle of incidence, and $m$ and $p$ are integers corresponding to the diffracted order of the anomaly. Here the grating axes are aligned with the Cartesian axes $(O x)$ and $(O y)$, and the plane of incidence is the $(z O x)$ plane. Note that in Eq. $1, m$ and $p$ do not play symmetrical roles, as the symmetry of the grating is broken by the incident light. In the range of wavelengths and periods covered in Fig. 2, the $( \pm 1,0),(0, \pm 1)$ and $(1, \pm 1)$ Rayleigh anomalies can be excited and their position, according to Eq. 1, is shown in Fig. 2 by solid white lines. Note that at normal incidence on a square grating, the $( \pm 1,0)$ and $(0, \pm 1)$ diffraction anomalies are degenerated and fall onto the same line. It clearly appears that the wavelength position of the resonance closely follows the position of the $( \pm 1,0)$ anomaly. In fact, careful analysis of Fig. 2 shows an anti-crossing between the $(1,0)$ Rayleigh mode and the LSPR for a period of about 430 $\mathrm{nm}$. A similar effect, although less prominent, occurs between the $(1,1)$ mode and the LSRP for a lattice period of about 600 $\mathrm{nm}$. This behavior corresponds to strong coupling between the LSP resonance sustained by the nanoparticles in the array and the Rayleigh anomalies, determined by their periodic arrangement. The hybridization of the LSP with the light scattered in the plane of the nanoparticles at the Rayleigh anomaly provides an opportunity to combine the high extinction cross-section of plasmonic modes with the narrow bandwidth of the Rayleigh anomalies to design spectral lineshapes. The sharp and Fanolike lineshape of the lattice mode is more clearly seen on the line cuts shown as inserts in Fig. 3 for a period of $500 \mathrm{~nm}$. The broader resonance around $\lambda=630 \mathrm{~nm}$ corresponds to the other hybrid mode.

More insight on the nature of both hybrid modes can be gained by plotting the normalized electric field at the wavelength corresponding to each of the modes, for different periods. Let us start with a period $D=300 \mathrm{~nm}$, corresponding to the very left part of Fig. 2 . The extinction spectrum shows only one relatively broad resonance around $\lambda=600 \mathrm{~nm}$. The field map associated with that resonance is shown in Fig. 3a and evidences that the electric field is mainly localized around the gold nanodisks, as expected for isolated particles. If the period is increased to $D=500 \mathrm{~nm}$, the extinction spectrum shows two resonances. The field map associated with the lower wavelength, broader resonance (Fig. 3b) evidences field maxima that are localized in the vicinity of the gold nanodisks, similarly to Fig. 3a and linked with the dipolar plasmonic resonance of the isolated nanodisk. On the other hand, the field map corresponding to the sharp resonance (Fig. 3c) evidences a simultaneous excitation of the metal nanoparticles along the polarization direction and a standing grazing wave (Rayleigh anomaly) in the perpendicular $(\mathrm{Oy})$ direction. In other words, the coupling gives birth to two hybrid modes, one with a more "photon-like", delocalized nature, and the other one exhibiting a "plasmon-like", localized nature. As the plasmon-like mode is more localized than the photon-like mode, it experiences more Joule losses resulting in a much broader peak with a low quality factor. This is further confirmed by the field maps computed for an array exhibiting a period of $650 \mathrm{~nm}$ (Fig. 3d-e). Fig. 3d corresponds to the plasmonic mode, and Fig. 3e to the photonic mode, evidencing coupling with the $(1,1)$ Rayleigh anomaly at $\pm 45^{\circ}$ for the axes of the array.

In order to underline the critical role played by the grating period, we computed the quality factor as a function of period for four different nanodisk diameters (Fig. 4a, note the log scale on the vertical axis). Note that in Fig. 4, the range of studied diameters and periods has been chosen to yield lattice modes in the visible and near-infrared spectrum. Extremely high values of $Q$ can be obtained $(Q>1000)$ for high values of the lattice period, while an isolated gold nanodisk would exhibit $Q \sim 5-10$. Interestingly, the value of $Q$ seems to continuously increase with the period. Also, higher values of $Q$ are observed 

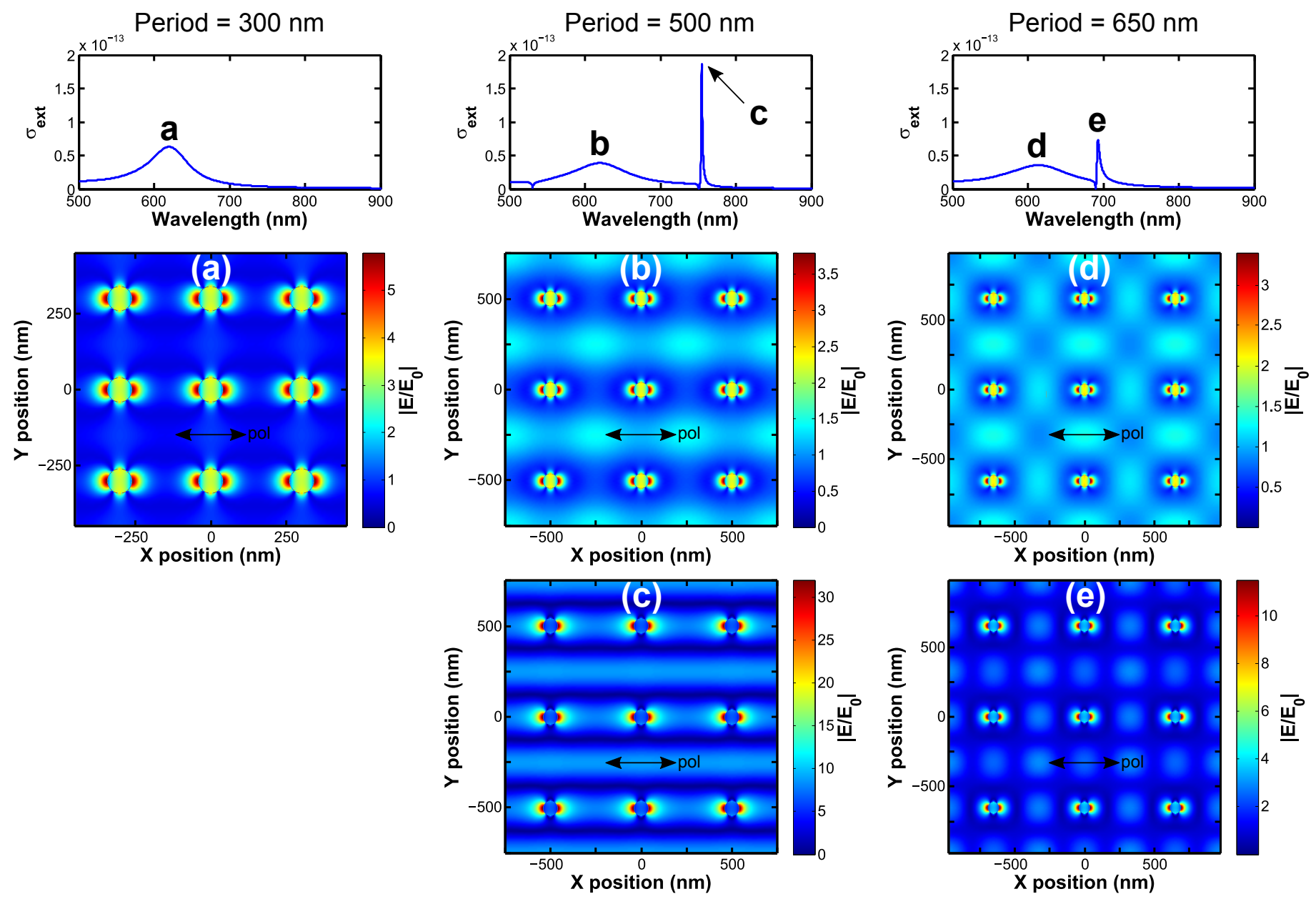

Fig. 3. Normalized electric field modulus maps for different lattice periods. The insets on the top of each of the columns are line cuts of Fig. 2, showing the extinction cross-section $\sigma_{\text {ext }}$ vs. wavelength for three different periods: $D=300$ (left column), $D=500$ $\mathrm{nm}$ (center column) and $D=650 \mathrm{~nm}$ (right column). The arrows indicate the wavelengths where the field maps have been calculated. (a) FDTD maps of the normalized electric field modulus computed in a plane located at mid-height of the metal nanoparticles, for an array with $D=300 \mathrm{~nm}$, at $\lambda=630 \mathrm{~nm}$. The black arrow denotes the incident polarization. The same field maps have been computed for: (b) $D=500 \mathrm{~nm}$ and $\lambda=630 \mathrm{~nm}$; (c) $D=500 \mathrm{~nm}$ and $\lambda=750 \mathrm{~nm}$; (d) $D=650 \mathrm{~nm}$ and $\lambda=630 \mathrm{~nm}$; (e) $D=650 \mathrm{~nm}$ and $\lambda=700 \mathrm{~nm}$.

for smaller nanodisk diameters. The former effect can be understood as follows. When $D$ increases, the lattice mode is getting closer to the position of Rayleigh anomaly (see Fig. 2). As a consequence, the hybrid mode becomes more and more "photonlike" in nature, and exhibits reduced losses. What will ultimately limit the value of the $Q$-factor is the size of the particles. As the period increases the spectral position of the lattice mode red-shifts, hence the nanoparticles are becoming smaller with respect to the resonance wavelength. This implies that their scattering efficiency, and thus the diffractive coupling strength between the LSP resonances near the Rayleigh anomaly, should decrease for higher values of the lattice period.

To evidence the influence of the dielectric environment, we also computed the quality factor as a function of the period in the case of an asymmetric environment (glass substrate and air superstrate). Results are presented in Fig. $4 \mathrm{~b}$ for different nanodisks diameters. Inside that range of periods, the observed behavior offers a striking difference with the symmetric case. Optimum values of the lattice period clearly appear that for each diameter, and this optimum value increases with the diameter of the nanodisks. Moreover, the obtained values of $Q$ are more than one order of magnitude lower than in the symmetric geometry. Value in the range $Q \sim 10-13$ are obtained, which is roughly three times the value for an isolated nanodisk. We can also note that in order to obtain an efficient linewidth reduction, larger particles than in the symmetric case have to be considered. In other words, if we set the grating period, the linewidth reduction will be efficient for different diameters in different dielectric environments. Altogether, from a "linewidth engineering" perspective, working in a homogeneous environment is clearly beneficial. However, a significant linewidth reduction can still be observed in an asymmetric environment, provided the grating is properly designed.

The role played by the dielectric environment can be qualitatively understood as follows. In the asymmetric geometry, the radiation pattern tends to be highly asymmetric with most of the light scattered inside the substrate [39]. This leads to a weak angular overlapping with grazing diffracted orders. When the dielectric environment is symmetric, so is the radiation pattern - allowing for a much higher coupling into in-plane scattered 

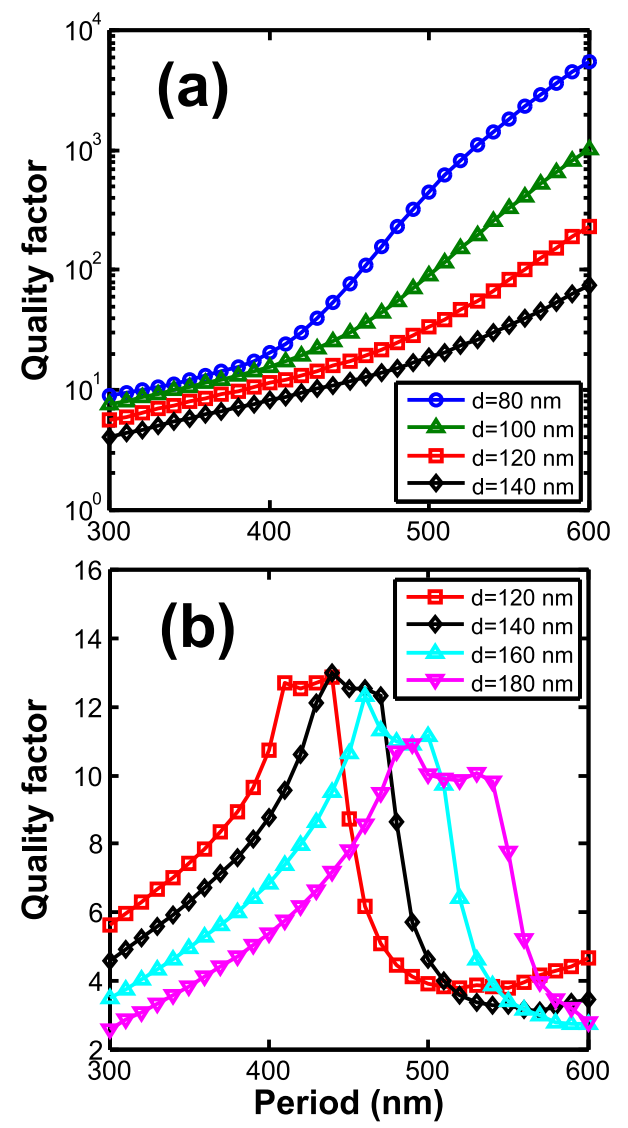

Fig. 4. Linewidth engineering using lattice modes. (a) Calculated quality factor of the lattice mode of arrays of gold nanodisks as a function of the lattice period, for different disk diameters, at normal incidence. The dielectric environment is symmetric $\left(n_{1}=n_{2}=1.5\right)$. (b) Same, with air as the superstrate $\left(n_{2}=1\right)$.

light. This is why setting an adapted period for the array is then a necessary, but not sufficient, condition to observe high- $Q$ lattice modes. Further evidence of the central role played by the radiative pattern of the nanoparticles will be given in Section 4 .

It must be underlined that while the symmetric configuration yields by far the sharpest resonances, such a configuration is not without drawbacks from an experimental perspective [23]. First, the nanoparticles are isolated from their environment, limiting potential interactions with emitters or analytes. But most importantly, as the effective refractive index around the nanoparticles is higher in this geometry, the lattice modes are red-shifted with respect to the asymmetric geometry. Obtaining high energy resonances with the symmetric geometry hence requires significantly smaller nanoparticles whose reliable fabrication is more demanding.

In the following we turn our attention on the experimental verification of our findings on gold and aluminum nanoparticles arrays.

\section{EXPERIMENTAL METHODS}

\section{A. Sample fabrication}

Arrays of gold and aluminum nanoparticles were made using electron beam lithography, which allows the realization of nanostructures with well-controlled size, shape and period [40].

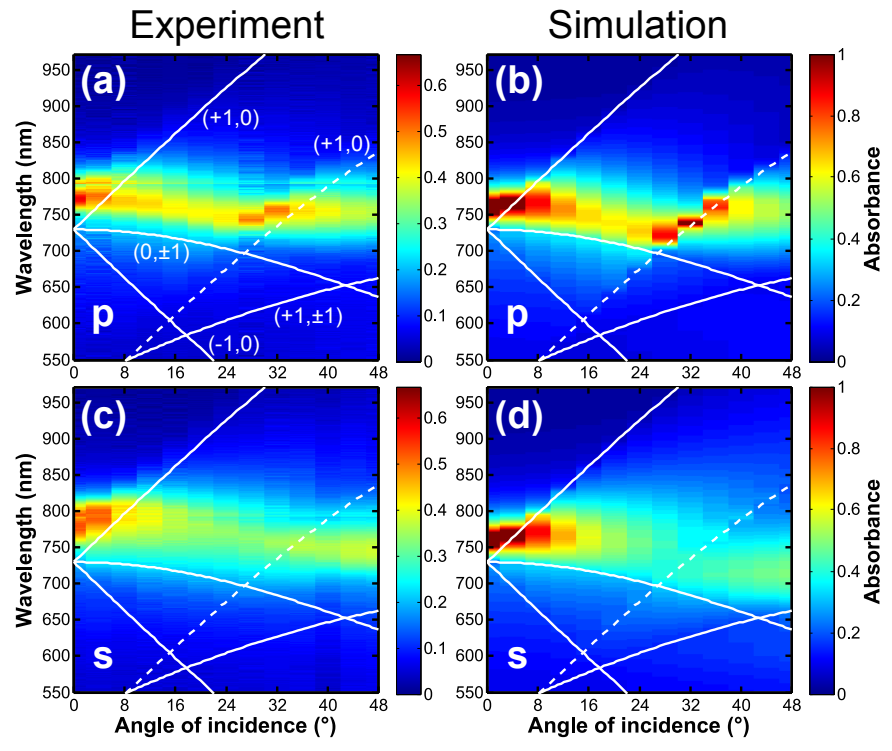

Fig. 5. Experimental $(a, c)$ and simulated $(b, d)$ dispersion curves of the gold nanodisks arrays for $s(\mathrm{a}, \mathrm{b})$ and $p(\mathrm{c}, \mathrm{d})$ polarizations. The period of the array is $D=480 \mathrm{~nm}$, the diameter of the nanodisks is $d=180 \mathrm{~nm}$. White lines represent the position of the Rayleigh anomalies computed using Eq. 1 with $n_{1}=1.52$ and $n_{2}=1.52$ (solid lines) or $n_{2}=1$ (dotted lines).

The gold nanoparticles are $50 \mathrm{~nm}$ thick nanodisks and were deposited onto a glass substrate with a $3 \mathrm{~nm}$ thick $\mathrm{TiO}_{2}$ layer to ensure adhesion of gold onto glass. Two different aluminum nanoparticle samples were made: $d=120 \mathrm{~nm}$ nanodisks exhibiting a LSP resonance in the visible and $d=60 \mathrm{~nm}$ nanodisks with a LSP in the UV spectrum. The aluminum thickness was set to $50 \mathrm{~nm}$ in both cases. Both structures were deposited onto fused silica to ensure transparency in the UV. Note that for aluminum no adhesion layer is required as this metal firmly adheres onto glass [41]. The total size of the arrays is $50 \mu \mathrm{m} \times 50 \mu \mathrm{m}$.

The quality of the fabricated structures was characterized with both atomic force and electron microscopy. Typical images are shown in Fig. 1b for gold nanodisks and Fig. 1c for $d=120 \mathrm{~nm}$ aluminum nanodisks. Regular arrays are observed in both cases, but clearly the gold nanoparticles exhibit a more defined shape than the aluminum ones. This is a well-known difference in the thermal evaporation of both metals, as it is much more difficult to obtain smooth surfaces of aluminum because it tends to nucleate in the early stages of deposition [42]. After measurement of the arrays' dispersion curves (see below), the nanostructures were spin-coated with PMMA to obtain the symmetric geometry. This ensures that the comparison between the asymmetric and symmetric geometries is made on the same arrays. The thickness of the deposited PMMA layer is $\sim 180 \mathrm{~nm}$. Let us underline that the refractive index of PMMA does not exactly match that of the glass substrate. However, as it is slightly lower, this ensures that no guided modes can propagate inside the PMMA layer and interact with the LSPR.

\section{B. Optical characterization}

The optical properties of all samples were measured using an angle-resolved extinction spectroscopy set-up. This setup allows the dispersion curves of the arrays to be retrieved, that is, the extinction plotted as a function of the angle of incidence 


\section{Experiment}
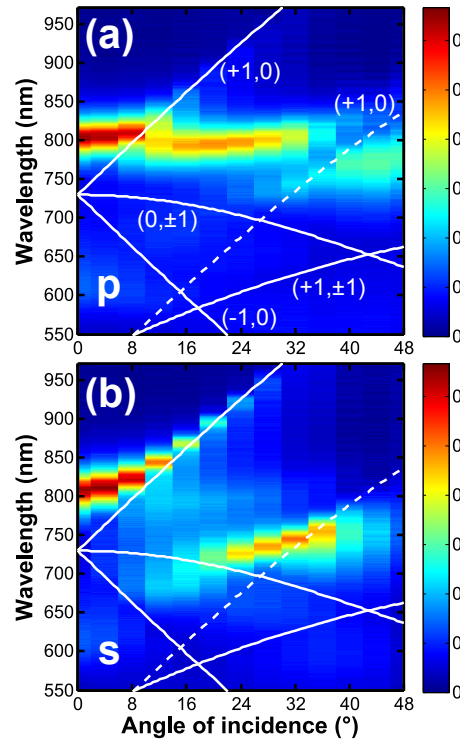
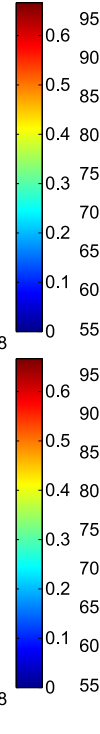

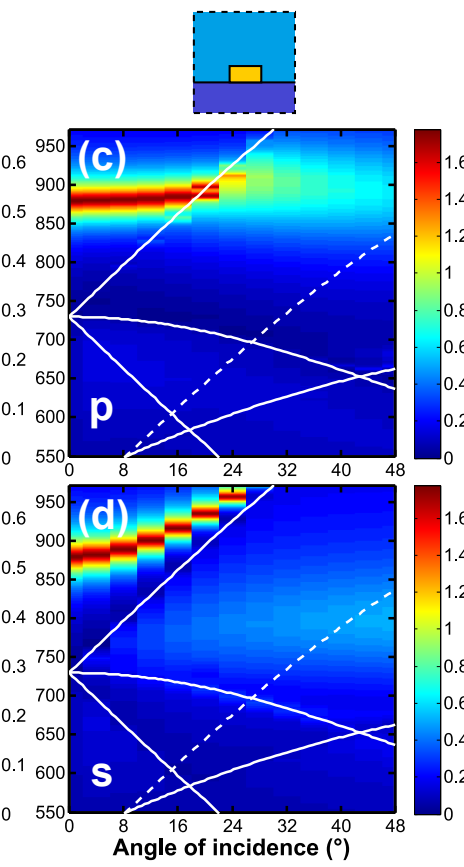

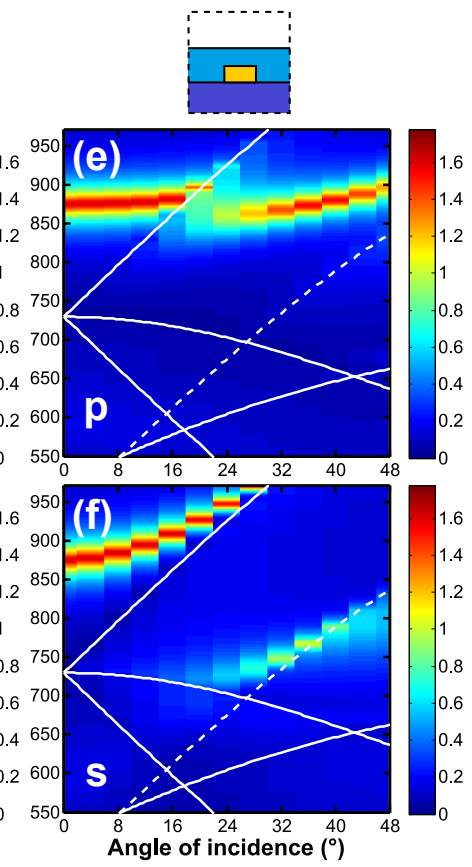

Fig. 6. Experimental (a-b) and simulated (c-h) dispersion curves of the PMMA-covered gold nanodisks arrays for $s$ and $p$ polarizations. The period of the array is $D=480 \mathrm{~nm}$, and the diameter of the nanodisks is $d=180 \mathrm{~nm}$. White lines represent the position of the Rayleigh anomalies computed using Eq. 1 with $n_{1}=1.52$ and $n_{2}=1.52$ (solid lines) or $n_{2}=1$ (dotted lines). The simulations were performed using three different geometries as pictured in the insets: (c-d) a semi-infinite PMMA superstrate; (e-f) a 180-nmthick PMMA layer onto the top of the substrate, $(\mathrm{g}-\mathrm{h})$ a 180-nm-thick PMMA standing onto the top of the nanoparticles.

and the wavelength (or, equivalently, the wavevector and the energy). The set-up works over a broad spectrum, from the UV to the near-infrared. Collimated light from a UV/visible lamp (Avalight-DHS, Avantes) is slightly focused onto the sample, which can be rotated by a controllable angle $\theta$ with regards to the incident beam. The light transmitted by the sample is collected using a microscope objective lens (MPlan UV 50x, Mitutoyo) confocally coupled to an optical fiber (solarisation resistant fused silica fiber, core diameter $100 \mu \mathrm{m}$ ) to define a collection area corresponding to the sample size. Finally, the output of the fiber is sent towards a spectrometer (IsoPlane SCT320, Princeton Instruments) equipped with a cooled camera (ProEM $1600^{2}$, Princeton Instruments). The polarization of the incident light is controlled by a broadband polarizer placed in the illumination path. To obtain the dispersion curves shown below, we measured an extinction spectrum for every $4^{\circ}$, from $\theta=0^{\circ}$ up to $\theta=48^{\circ}$. Note that in the following, the arrays are illuminated from the glass substrate and the angle of incidence used as the horizontal axis is measured in air (i.e. before refraction inside the substrate).

\section{GOLD NANOPARTICLE ARRAYS}

Fig. 5 presents the dispersion curves of an array of gold nanoparticles for s-polarization (electric field parallel to the $y$ axis) and $p$-polarization (electric field in the $x z$-plane) in the asymmetric geometry, that is, for gold nanoparticles in air on a glass substrate. In the left panels are plotted the experimental results, while the right panels show the corresponding FDTD simulations. On Fig. 5 are also plotted the position of the Rayleigh anomalies, according to Eq. 1. The two polarizations exhibit slightly different behaviors. The $p$-polarization shows a broad and relatively "flat" dispersion branch corresponding to the plasmon resonance of this branch, while in s-polarization an interaction with the $(1,0)$ glass / air Rayleigh anomaly appears. The agreement with the numerical simulations is very good. The most important difference between $p$ and s-polarizations concerns the $(1,0)$ glass/air anomaly (dotted white line in Fig. 5). In s-polarization, the anomaly has a detrimental effect on the plasmon branch, weakening its intensity for high angles (see Fig. $5 d$ ) while in $p$-polarization the anomaly seems to increase the absorbance. However, globally the effect of the diffraction anomalies is weak, confirming what we saw in Section 2: to obtain significant hybridization, a symmetric dielectric environment is required.

Next, we consider the case of the "symmetric" dielectric environment, i.e. after deposition of a $\sim 180 \mathrm{~nm}$ layer of PMMA onto the previously measured array. While such an environment cannot be considered as rigorously homogeneous due to the slight refractive index difference between glass $(n=1.52)$ and PMMA ( $n=1.49$ ), this configuration is close enough to the one discussed in Section 2. Experimental results are reported in Fig. $6 \mathrm{a}$ for $p$-polarisation and Fig. $6 \mathrm{~b}$ for $s$-polarization. Comparison with Fig. 5 shows a clear effect of the change in the dielectric environment. Notably, for s-polarization a clear bending of the LSP branch is observed as it approaches the $(1,0)$ symmetric Rayleigh anomaly. The resulting lattice mode presents a sharper profile: the quality factor of the lattice mode is found to be $Q=15$ at normal incidence, increasing to $Q=28$ at $\theta=12^{\circ}$. This is a clear demonstration of the beneficial influence of generating lattice modes inside a symmetric dielectric environment.

More insight can be gained by comparing the two polarizations. Considering Fig. $6 a$, it is remarkable that the $(+1,0)$ anomaly (corresponding to a grazing wave propagating along the $x$ direction) crosses the main dispersion branch with limited 
perturbation. In contrast, in the other polarization a strong interaction between the same anomaly and the LSPR is evidenced (Fig. 5b), with a bending of the band and the appearance of a Fano lineshape similar to those of Fig. 3. In other words, to be significantly active and hence allow the creation of lattice modes with sensible coupling, an anomaly must be coupled in a direction perpendicular to the incident polarization. This effect can be explained qualitatively by considering how a single nanoparticle scatters light. If we assume the nanoparticle to behave as a dipole, then its far-field radiation will be perpendicular to the induced polarization, which is along the polarization of the incident electric field. In other words, as each nanoparticle scatters light in a direction perpendicular to the incident polarization, it will preferentially couple with grazing diffractive orders along that direction. This can be ascertained by looking again at Fig. 3c, showing the electric field map at the wavelength corresponding to the excitation of a lattice mode. While the polarization is horizontal in the figure, it excites a grazing wave in the vertical direction. It is interesting to note that for non-square gratings, it implies that the position of the Rayleigh anomaly is governed by the period along the direction perpendicular to the incident polarization.

The bending of the plasmonic branch happens after interaction with the symmetric $(1,0)$ Rayleigh anomaly. In principle, in a homogeneous dielectric environment the asymmetric glass/air anomalies should not affect the LSP resonance. However an effect can be seen on the experimental measurements for both polarizations (dotted line in 6a-b). To understand this unintuitive behavior, we performed additional numerical simulations. In Fig. $6 \mathrm{c}$ and $6 \mathrm{~d}$ are plotted the simulated dispersion curves for a glass substrate $\left(n_{1}=1.52\right)$ covered with a semi-infinite PMMA superstrate $\left(n_{2}=1.49\right)$. A number of discrepancies with the measurements are obvious: the position of the LSP branch is red-shifted, and the high-angle behavior is totally different, especially in s-polarization where the secondary branch, experimentally observed, is not predicted by the simulations. In order to better match the experimental geometry, we performed additional simulations by replacing the semi-infinite superstrate by a finite-thickness PMMA layer. Results are presented in Fig. 6e-f. It can be seen that now in s-polarization a branch around the $(1,0)$ glass/air anomaly is visible. However there is still a significant red-shift in the position of the resonance when compared to measurements. Such a shift could be explained if the nanoparticles are experiencing a lower effective refractive index in the experimental configuration, for instance in the case of the presence of air inclusions in the polymer film. To check the validity of this hypothesis, we assumed that, when spin-coated the polymer did not perfectly fill the space between nanoparticles, but rather formed a film on top of the nanoparticles, with air between each nanoparticle. Results are shown on Fig. 6g-h, evidencing a good agreement with the experiments for both polarizations: the resonance position is close to the experimental one.

Now we can sum up our results concerning the effects of both polarization and dielectric environment into the following rule of thumb: the coupling strength between LSPs near the Rayleigh anomalies is mainly governed by the radiation pattern of the single scatterers, and how it spatially and angularly overlaps with the grazing diffractive orders. More precisely, the Fano splitting (i.e., the coupling strength) is governed by three physical parameters: (i) the spectral overlap between the initial modes, (ii) modal (i.e., spatial) overlap between the initial modes, and (iii) the initial linewidth.
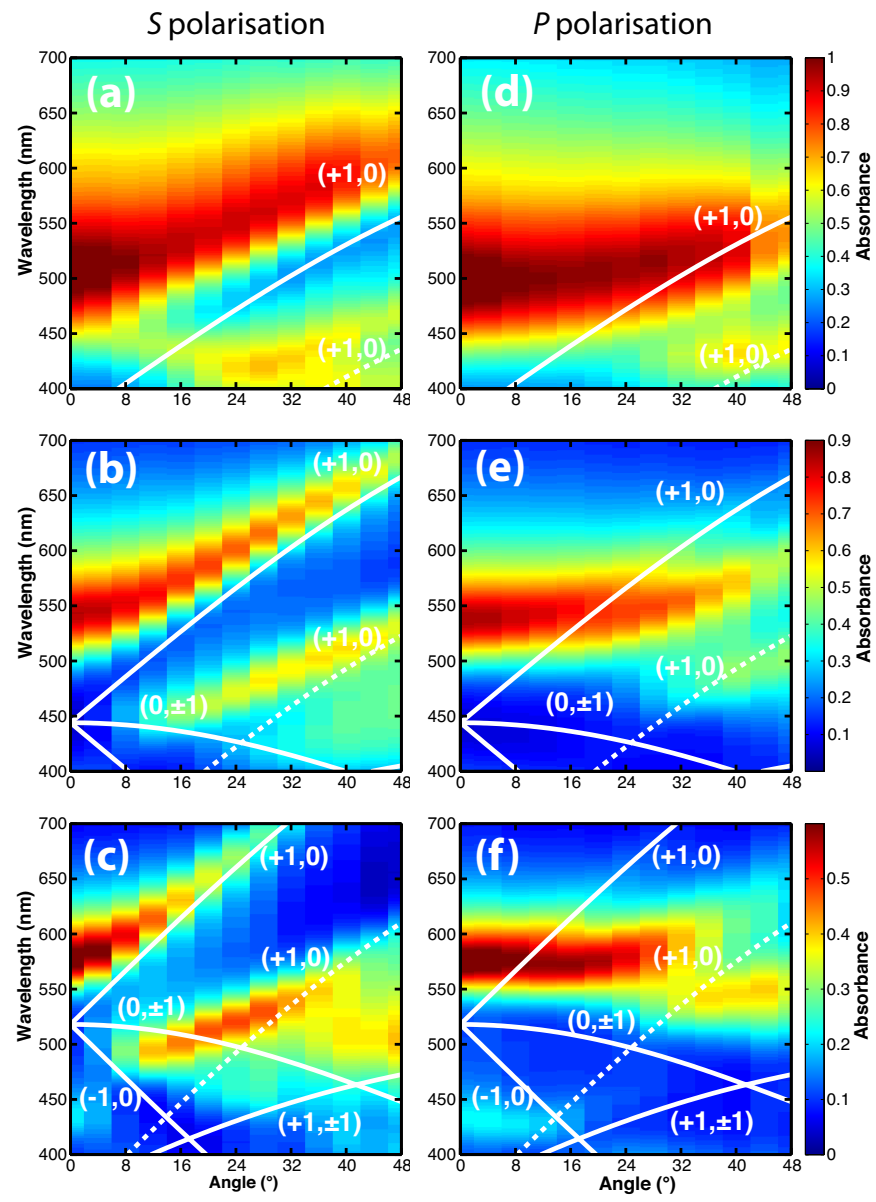

Fig. 7. Experimental dispersion curves of PMMA-covered aluminum nanodisk arrays for: (a-c) s-polarization, and (d-f) $p$ polarization. The diameter of the nanoparticles is $d=120 \mathrm{~nm}$. The period of the array is: $(\mathrm{a}, \mathrm{d}) D=250 \mathrm{~nm}$; (b,e) $D=300 \mathrm{~nm}$; and (c,f) $D=350 \mathrm{~nm}$. White lines represent the position of the glass/glass (solid lines) or glass/air (dotted lines) Rayleigh anomalies computed using Eq. 1.

\section{ALUMINUM NANOPARTICLE ARRAYS}

In this Section we apply our previous findings to the LSP sustained by aluminum nanoparticle arrays. As pointed out in the introduction, aluminum has a plasmonic response extending over a broad spectral region but the amount of losses, relative to gold, increases significantly for energies lower than the UV. Here, we discuss the possibility of using lattice resonances to reduce the amount of losses in two different spectral ranges: the first sample is designed to work in the visible range, the second sample is designed for the blue-UV range.

\section{A. Lattice modes in the visible}

The first sample has been designed to exhibit resonances in the visible spectrum. We fabricated arrays of aluminum nanodisks with a diameter of $120 \mathrm{~nm}$ and three different periods: $D=$ 250, 300 and $350 \mathrm{~nm}$. Since a symmetric dielectric environment yields the sharpest resonances, the sample was covered with PMMA. The measured dispersion curves are presented in Fig. 7 for both polarizations. As the diameter of the metallic nanoparticles is kept constant while the period is changing, we are effectively moving the spectral position of the Rayleigh anoma- 


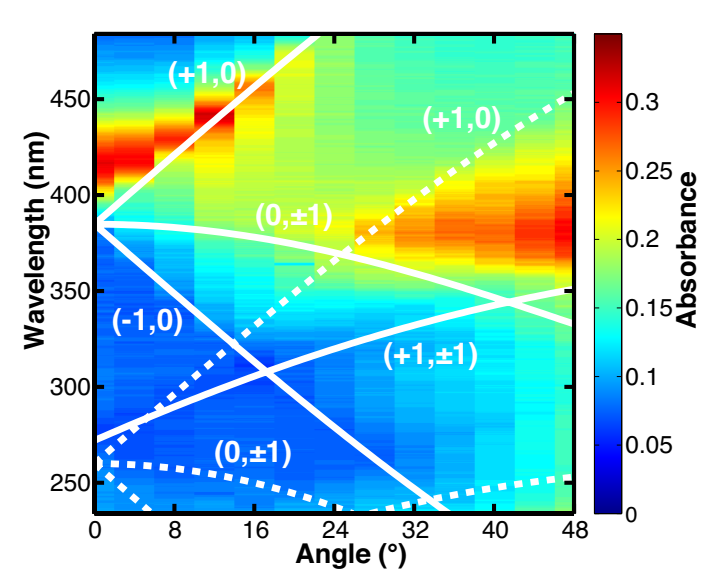

Fig. 8. Experimental dispersion curve of the $d=60 \mathrm{~nm}$ aluminum nanodisk arrays in the ultraviolet for $s$-polarization. The period of the array is $D=260 \mathrm{~nm}$. White lines represent the position of the glass/glass (solid lines) or glass/air (dotted lines) Rayleigh anomalies computed using Eq. 1.

lies for a fixed LSP resonance. The effect is clearly seen for $s$ polarization comparing Fig. 7a, b, and c. In Fig. 7a the anomaly is located far from the LSP, and a weak interaction is observed with the broad LSP resonance. At normal incidence the observed linewidth is quite similar to the one of an isolated aluminum nanoparticle $(Q \simeq 2.5$ ). When the period is increased to $D=300 \mathrm{~nm}$, the position of the $(1,0)$ Rayleigh anomaly shifts toward higher wavelengths and a stronger coupling of the LSP resonances is observed as a bending of the plasmon branch and linewidth reduction when the angle of incidence is increased. At normal incidence, the linewidth is significantly lower than previously $(Q \simeq 5$ ) and the resonance further sharpens when the angle of incidence increases $\left(Q \simeq 9.5\right.$ for $\left.\theta=32^{\circ}\right)$. Finally, for $D=350 \mathrm{~nm}$, the anomaly is very close to the LSP resonance, leading to a stronger coupling and the apparition of a sharper lattice mode $(Q \simeq 9$ at normal incidence). For $p$-polarization (Fig. 7d-e), we obviously observe the same linewidth at normal incidence due to the symmetry of the nanoparticles, but the angular dependence is different, as no bending and linewidth reduction are observed when $\theta$ increases. This is due to the weak coupling efficiency between the LSPs excited in $p$-polarization near the $(1,0)$ Rayleigh anomaly. We also note that similarly to what happened with gold nanoparticle arrays, the glass/air Rayleigh anomaly influences the dispersion curve (see Fig. 7e), while it should be absent in a homogeneous environment. As previously, this is likely due to the finite thickness of the PPMA layer and the presence of air inclusions.

The obtained quality factors can be compared to the quality factor for isolated $\mathrm{Al}$ nanoparticles, which is generally $Q \approx 3-4$ in the visible range [5]. Hence, the linewidth engineering enabled by lattice modes allows $Q$-factors to be reached which are comparable to isolated gold nanoparticles in the visible. It is also interesting to compare Fig. $7 \mathrm{~b}$ and $7 \mathrm{c}$. In the latter, the anomaly is very close to the LSP spectral position, leading to a sharp resonance at normal incidence whose intensity dramatically decreases when the angle of incidence increases. In contrast, in Fig. $7 \mathrm{~b}$, the normal incidence linewidth is higher (due to the higher frequency mismatch between the LSP and the anomaly) but as the angle is increased the resonance sharp- ens without being too detrimental to the lattice mode intensity. Consequently, if sharp lattice modes at normal incidence are sought, then the Rayleigh anomaly should be designed to appear as close as possible from the LSP resonance. But if angular tolerance is required, then a minimal spectral mismatch should be introduced between the anomaly and the LSP.

\section{B. Lattice modes in the blue-UV}

Finally, we present results obtained on aluminum nanodisk arrays that have been designed to exhibit a LSP resonance in the blue and near-UV region. As explained in the introduction, aluminum can sustain LSP resonances down to deep-UV wavelengths, however the particle diameter is a strong practical limitation. We made $d=60 \mathrm{~nm}$ diameter nanoparticles as a trade-off between exhibiting UV resonances and fabrication constraints. The period of the array is $D=260 \mathrm{~nm}$. We chose the superstrate to be air to avoid any red-shift of the plasmon resonance with the dielectric environment.

The experimental dispersion curve for s-polarization is presented in Fig. 8. A clear interaction between the LSP resonance around $\lambda=420 \mathrm{~nm}$ and scattered light near the $(1,0)$ Rayleigh anomaly is observed, leading to hybridization and the appearance of a sharper resonance. Under normal incidence, we obtain $Q \simeq 8$, a value that should be compared to the $Q \approx 4-5$ obtained for isolated aluminum particles [5].

\section{CONCLUSION}

In summary, we have reported a study of the lattice modes, arising from diffractive coupling between localized plasmon modes sustained by individual nanoparticles near Rayleigh anomalies of the periodic array. Our results evidenced the crucial role played by the dielectric environment and the incident polarization on the coupling strength and subsequent formation of lattice modes. It was shown that the hybridization between the localized plasmon resonances and the formation of the lattice modes is mainly controlled by the radiation pattern of the individual metal scatterers forming the arrays. If carefully designed, nanoparticles arrays can exhibit lattice modes with remarkably high quality factors. For arrays of gold nanoparticles, it is possible to reach $Q$-factors of several hundreds. In the case of aluminum nanoparticles used in the visible spectrum, $Q$-factors of the same magnitude as those obtained for isolated gold nanoparticles were obtained, in spite of the relatively higher intrinsic losses of $\mathrm{Al}$ compared to gold. Moreover, it should be noted that the quality of the lithographed aluminum nanostructures was lower than the gold ones, which might have played a detrimental role on the $Q$-factor. As mentioned earlier, this is mainly due to the surface roughness of the evaporated $\mathrm{Al}$ film, which is difficult to master [42]. However this limit can be lifted by using, for instance, the newly developed epitaxial aluminum films [43] to obtain high-quality $\mathrm{Al}$ nanostructures exhibiting low scattering and absorption losses. Altogether, we believe our results are a further demonstration of the potential of aluminum as a broadband plasmonic material.

\section{FUNDING INFORMATION}

CNRS and The Royal Society joint grant "Alu3D". DK is supported by Région Champagne-Ardenne. Financial support of Nano'Mat (www.nanomat.eu) by the "Ministère de 
l'enseignement supérieur et de la recherche", the "Conseil régional Champagne-Ardenne", the "FEDER fund" and the "Conseil général de l'Aube" is acknowledged. This work was also partially supported by the HPC Center of Champagne-Ardenne ROMEO. W.P.W., W.D. and A.V.Z. work is supported by EPSRC (UK). A.V.Z. acknowledges support from the Royal Society and the Wolfson Foundation.

\section{ACKNOWLEDGMENTS}

This work was performed during D.G.'s stay at King's College London as a Visiting Senior Research Fellow, thanks to support from the CNRS and the Région Champagne Ardenne. We are most grateful to Loïc Le Cunff for his help with FDTD calculations. The data access statement: all the data supporting this research are presented in full in the results section and supplementary materials.

\section{REFERENCES}

1. A. V. Zayats, I. I. Smolyaninov, and A. A. Maradudin, "Nano-optics of surface plasmon polaritons," Phys. Rep. 408, 131-314 (2005).

2. P. West, S. Ishii, G. Naik, N. Emani, V. Shalaev, and A. Boltasseva, "Searching for better plasmonic materials," Laser \& Photon. Rev. 4, 795808 (2010).

3. D. Gérard and S. K. Gray, "Aluminium plasmonics," J. Phys. D: Appl. Phys. 48, 184001 (2015).

4. F. Bisio, R. P. Zaccaria, R. Moroni, G. Maidecchi, A. Alabastri, G. Gonella, A. Giglia, L. Andolfi, S. Nannarone, L. Mattera, and M. Canepa, "Pushing the high-energy limit of plasmonics," ACS Nano 8, 9239-47 (2014).

5. J. Martin, M. Kociak, Z. Mahfoud, J. Proust, D. Gérard, and J. Plain, "High-resolution imaging and spectroscopy of multipolar plasmonic resonances in aluminum nanoantennas," Nano Lett. 14, 5517-23 (2014).

6. Q. Zhang, G. Li, X. Liu, F. Qian, Y. Li, T. C. Sum, C. M. Lieber, and $Q$. Xiong, "A room temperature low-threshold ultraviolet plasmonic nanolaser," Nat. Commun. 5, 5953 (2014).

7. S. J. Tan, L. Zhang, D. Zhu, X. M. Goh, Y. M. Wang, K. Kumar, C.-W. Qiu, and J. K. W. Yang, "Plasmonic color palettes for photorealistic printing with aluminum nanostructures," Nano Lett. 14, 4023-9 (2014).

8. J. Olson, A. Manjavacas, L. Liu, W.-S. Chang, B. Foerster, N. S. King, M. W. Knight, P. Nordlander, N. J. Halas, and S. Link, "Vivid, fullcolor aluminum plasmonic pixels," Proc. Natl. Acad. Sci. 111, 14348-53 (2014).

9. B. Y. Zheng, Y. Wang, P. Nordlander, and N. J. Halas, "Color-selective and CMOS-compatible photodetection based on aluminum plasmonics," Adv. Mater. 26, 6318-6323 (2014).

10. M. B. Ross, C. A. Mirkin, and G. C. Schatz, "Optical properties of one, two-, and three-dimensional arrays of plasmonic nanostructures," J. Phys. Chem. C 120, 816-830 (2016).

11. N. Bonod and J. Neauport, "Diffraction gratings: from principles to applications in high-intensity lasers," Adv. Opt. Photonics 8, 156-199 (2016).

12. V. Giannini, Y. Francescato, H. Amrania, C. C. Phillips, and S. A. Maier, "Fano resonances in nanoscale plasmonic systems: a parameter-free modeling approach," Nano Lett. 11, 2835-40 (2011).

13. B. Gallinet and O. J. F. Martin, "Relation between near-field and far-field properties of plasmonic Fano resonances," Opt Express 19, 22167-75 (2011).

14. A. Väkeväinen, R. Moerland, H. Rekola, A.-P. Eskelinen, J.-P. Martikainen, D.-H. Kim, and P. Törmä, "Plasmonic surface lattice resonances at the strong coupling regime," Nano Lett. 14, 1721-1727 (2013).

15. M. Meier, A. Wokaun, and P. Liao, "Enhanced fields on rough surfaces: dipolar interactions among particles of sizes exceeding the Rayleigh limit," J. Opt. Soc. Am. B 2, 931-949 (1985).

16. K. T. Carron, W. Fluhr, M. Meier, A. Wokaun, and H. W. Lehmann,
"Resonances of two-dimensional particle gratings in surface-enhanced raman scattering," J. Opt. Soc. Am. B 3, 430-440 (1986).

17. S. Zou, N. Janel, and G. C. Schatz, "Silver nanoparticle array structures that produce remarkably narrow plasmon lineshapes," J. Chem. Phys. 120, 10871-10875 (2004).

18. N. Félidj, G. Laurent, J. Aubard, G. Lévi, A. Hohenau, J. R. Krenn, and F. R. Aussenegg, "Grating-induced plasmon mode in gold nanoparticle arrays," J. Chem. Phys. 123, 221103 (2005).

19. B. Auguie and W. L. Barnes, "Collective resonances in gold nanoparticle arrays," Phys. Rev. Lett. 101, 143902 (2008).

20. V. Giannini, G. Vecchi, and J. Gómez Rivas, "Lighting up multipolar surface plasmon polaritons by collective resonances in arrays of nanoantennas," Phys. Rev. Lett. 105, 266801 (2010).

21. A. I. Kuznetsov, A. B. Evlyukhin, M. R. Gonçalves, C. Reinhardt, A. Koroleva, M. L. Arnedillo, R. Kiyan, O. Marti, and B. N. Chichkov, "Laser fabrication of large-scale nanoparticle arrays for sensing applications," ACS Nano 5, 4843-9 (2011).

22. W. Zhou, M. Dridi, J. Suh, C. Kim, M. Wasielewski, G. C. Schatz, and T. W. Odom, "Lasing action in strongly coupled plasmonic nanocavity arrays," Nature Nanotech 8, 506-511 (2013).

23. A. Nikitin, T. Nguyen, and H. Dellaporta, "Narrow plasmon resonances in diffractive arrays of gold nanoparticles in asymmetric environment: Experimental studies," Appl. Phys. Lett. 102, 221116 (2013).

24. J. Marae-Djouda, R. Caputo, N. Mahi, G. Lévêque, A. Akjouj, P.-M. Adam, and T. Maurer, "Angular plasmon response of gold nanoparticles arrays: approaching the rayleigh limit," Nanophotonics (2016).

25. G. Vecchi, V. Giannini, and J. Gómez Rivas, "Shaping the fluorescent emission by lattice resonances in plasmonic crystals of nanoantennas," Phys. Rev. Lett. 102, 146807 (2009).

26. R. Czaplicki, A. Kiviniemi, J. Laukkanen, J. Lehtolahti, M. Kuittinen, and M. Kauranen, "Surface lattice resonances in second-harmonic generation from metasurfaces," Opt. Lett. 41, 2684 (2016).

27. A. Yang, T. B. Hoang, M. Dridi, C. Deeb, M. H. Mikkelsen, G. C. Schatz, and T. W. Odom, "Real-time tunable lasing from plasmonic nanocavity arrays," Nat Comms 6:6939, doi:10.1038/ncomms7939 (2015).

28. G. Lozano, D. J. Louwers, S. R. K. Rodríguez, S. Murai, O. T. A. Jansen, M. A. Verschuuren, and J. Gómez Rivas, "Plasmonics for solidstate lighting: enhanced excitation and directional emission of highly efficient light sources," Light: Science \& Applications 2, e66 (2013).

29. G. Lozano, G. Grzela, M. A. Verschuuren, M. Ramezani, and J. Gómez Rivas, "Tailor-made directional emission in nanoimprinted plasmonic-based light-emitting devices," Nanoscale 6, 9223-9 (2014).

30. F. Liu and X. Zhang, "Fano coupling between rayleigh anomaly and localized surface plasmon resonance for sensor applications," Biosensors and Bioelectronic 68, 719-725 (2015).

31. F. J. García de Abajo, J. J. Sáenz, I. Campillo, and J. S. Dolado, "Site and lattice resonances in metallic hole arrays," Opt Express 14, 7-18 (2006).

32. F. J. García de Abajo, "Colloquium: Light scattering by particle and hole arrays," Rev. Mod. Phys. 79, 1267-1290 (2007).

33. M. Kataja, T. K. Hakala, A. Julku, M. J. Huttunen, S. van Dijken, and P. Törmä, "Surface lattice resonances and magnetooptical response in magnetic nanoparticle arrays," Nat Comms 6, doi:10.1038/ncomms8072 (2015).

34. M. Laroche, S. Albaladejo, R. Gómez-Medina, and J. J. Sáenz, "Tuning the optical response of nanocylinder arrays: An analytical study," Phys. Rev. B 74, 245422 (2006).

35. Lumerical Solutions, FDTD Solutions.

36. E. D. Palik, ed., Handbook of Optical Constants of Solids (Academic Press, 1985).

37. P. B. Johnson and R. W. Christy, "Optical constants of the noble metals," Phys. Rev. B 6, 4370-4379 (1972).

38. A. Aminian and Y. Rahmat-Samii, "Spectral FDTD: A novel technique for the analysis of oblique incident plane wave on periodic structures," IEEE Trans. Antennas Propag. 54, 1818 (2006).

39. M. A. Lieb, J. Zavislan, and L. Novotny, "Single-molecule orientations determined by direct emission pattern imaging," J. Opt. Soc. Am. B 21, 1210-1215 (2004). 
40. G. Lérondel, S. Kostcheev, and J. Plain, "Nanofabrication for plasmonics," in "Plasmonics," , vol. XVI of Springer Series in Optical Sciences, S. Enoch and N. Bonod, eds. (Springer, Berlin, Germany, 2012), pp. 269-316.

41. J. Martin, J. Proust, D. Gérard, and J. Plain, "Localized surface plasmon resonances in the ultraviolet from large scale nanostructured aluminum films," Opt. Mater. Express 3, 954-959 (2013).

42. J. Martin and J. Plain, "Fabrication of aluminium nanostructures for plasmonics," J. Phys. D: Appl. Phys. 48, 184002 (2015).

43. F. Cheng, P.-H. Su, J. Choi, S. Gwo, X. Li, and C.-K. Shih, "Epitaxial growth of atomically smooth aluminum on silicon and its intrinsic optical properties," ACS Nano 10, 9852-9860 (2016). 